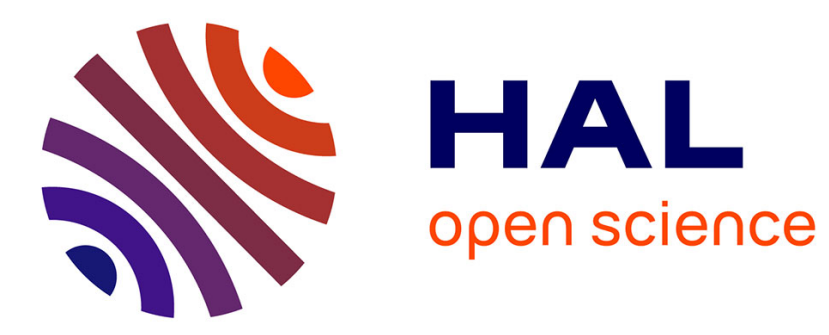

\title{
Analysis of Chaos Time Domain Reflectometry for the Soft Fault Detection in a Cable
}

I Bzikha, P Monferran, V Velayudhan, Alain Reineix

\section{To cite this version:}

I Bzikha, P Monferran, V Velayudhan, Alain Reineix. Analysis of Chaos Time Domain Reflectometry for the Soft Fault Detection in a Cable. EMC Europs Symposium, Sep 2020, Rome - Virtual Conference, Italy. 10.1109/EMCEUROPE48519.2020.9245836 . hal-03348648

\section{HAL Id: hal-03348648 \\ https://hal.science/hal-03348648}

Submitted on 19 Sep 2021

HAL is a multi-disciplinary open access archive for the deposit and dissemination of scientific research documents, whether they are published or not. The documents may come from teaching and research institutions in France or abroad, or from public or private research centers.
L'archive ouverte pluridisciplinaire HAL, est destinée au dépôt et à la diffusion de documents scientifiques de niveau recherche, publiés ou non, émanant des établissements d'enseignement et de recherche français ou étrangers, des laboratoires publics ou privés. 


\section{Analysis of Chaos Time Domain Reflectometry for the Soft Fault Detection in a Cable}

\author{
I. Bzikha \\ Xlim laboratory \\ Limoges, France \\ ihssane.bzikha@xlim.fr
}

\author{
P. Monferran \\ Xlim laboratory \\ Limoges, France \\ paul.monferran@xlim.fr \\ A. Reineix \\ Xlim laboratory \\ Limoges, France \\ alain.reineix@xlim.fr
}

\author{
V. Velayudhan \\ Xlim laboratory \\ Limoges, France \\ vipin.velayudhan@xlim.fr
}

\begin{abstract}
This paper proposes to analyze the performance in terms of soft fault detection of two chaotic signals using the chaos time domain reflectometry. These signals which are 1) a combination of the logistic map and Bernoulli map and 2) the Lorenz signal are depicted in order to remind their usage limitations. Although this paper demonstrates the relevance of the proposed chaotic signals for the soft fault detection, we propose also to compare the performances of each signal with a statistical approach. The study leads to conclude than the combination of the logistic map and Bernoulli map is more reliable than the Lorenz signal.
\end{abstract}

Index Terms-chaos time domain reflectometry (CTDR), soft fault detection, chaotic signals comparison

\section{INTRODUCTION}

Cables are still one of the most used process for the energy or information transfer despite the wireless development. Strong environmental aggressions can degrade cables and corrupt the transmitted signal [1]. Therefore, the fault detection was becoming an important challenge in the industry as, for instance, aeronautic or telecommunications [2], [3]. Hence several methods [4]-[6] have been developed in order to detect the faults, and, in particular their locations. Most of them are based on the reflectometry process which is a method which has proven its efficiency in terms of fault detection. The reflectometry principle is based on the propagation of an injected signal along the cable and the analysis of the reflected signal which contains the information related to each impedance discontinuity (load, junction, connector, fault) [7].

Among these reflectrometry-based methods, the chaos time domain reflectometry (CTDR) method has been less studied. In terms of fault detection, a method has been proposed in [8]. The chaotic signal is generated using a Colpitts oscillator. Indeed, it has been demonstrated than using a specific input current, this oscillator can produce an output behavior. Measurements with different hard and soft faults leads to good results for the location of open circuits, short circuits, impedance discontinuities and other different damage on wires. Simulations have been also perform in order to prove the ability for real time diagnosis. This method is quite efficient for single cable topology, however, the oscillators must be well designed in order to propose a chaotic behavior. Moreover, this chaotic behavior cannot be managed as it depends on the oscillator design. As a further matter, an average measurement from 1000 measures must be performed in order to reduce the noise of the test bench. Thus, another method has been proposed in [9], [10]. In [10], it has been demonstrated that a combination between the logistic map and the Bernoulli map leads to a chaotic behavior "choosing properly" the parameters. Moreover, the orthogonality of the generated chaotic signals has been shown modifying slightly the parameters. Hence, simulations highlight the ability of the hard fault detection for complex wire network. Thus, this chaotic signal is used in [9] to demonstrate the efficiency of the method in a high noisy cable network, and, for real time measurements. From a measurement bench where the chaotic signal is generated with an arbitrary wave generator, the sensitivity analysis carried out in this article was promising for the soft fault detection.

In this paper, we focus on soft fault detection for a simple coaxial cable. We propose to compare the efficiency of two chaotic signals (the one used in [9] and the Lorenz signal) in terms of soft fault detection. Both signals have been still studied separately. We propose here to compare their performance for the soft fault detection. Hence, a simple cable modeling is proposed. Thus, we build a code in the time domain based on the transmission line theory in order to analyze the reflected signal. Then, a sensitivity analysis is carried out from simulations in order to compare the efficiency of two chaotic signals in terms of soft fault detection.

First, the principle of the time domain code and the wire fault modeling are quickly reminded in section II. In section III, the combination of the logistic map and Bernoulli map is depicted and a parametric study is carried out in terms of fault detection. The same study is performed in section IV for the Lorenz signal. Then, a comparison of the performance of each signal for the fault detection is proposed in section $\mathrm{V}$. 


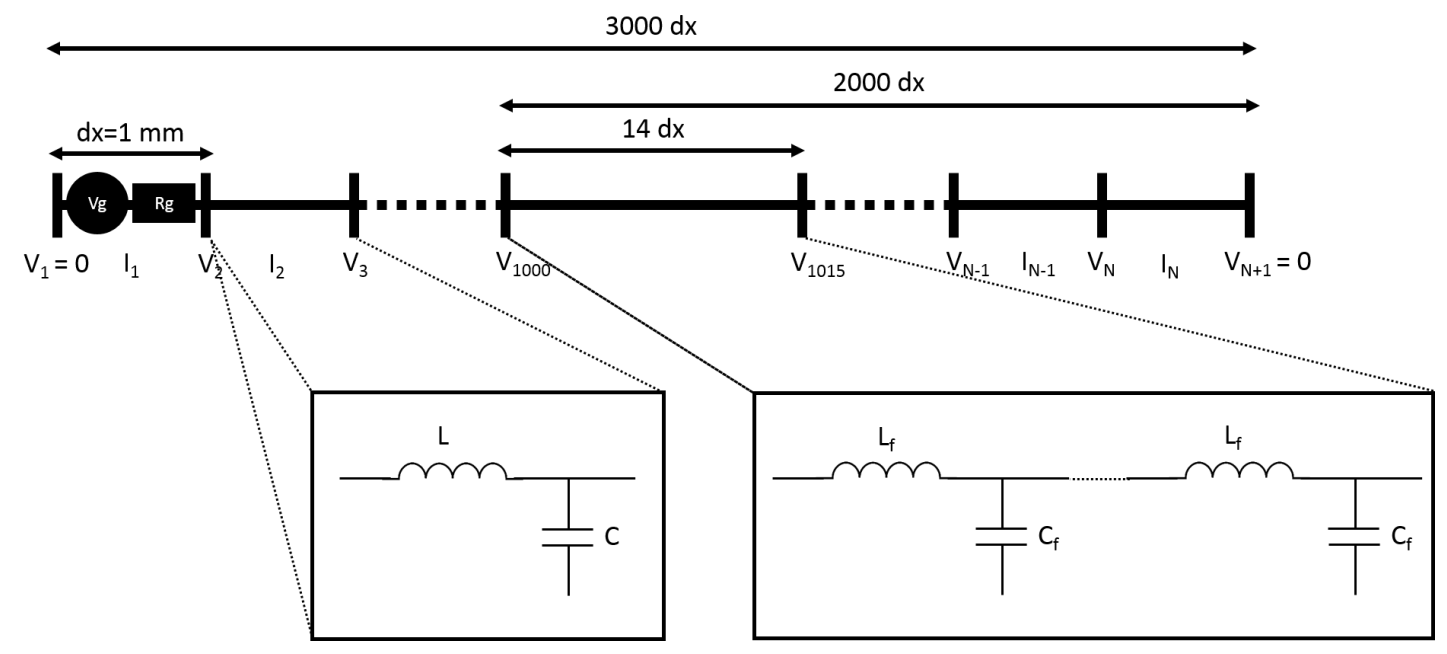

Fig. 1. Transmission line model of the coaxial cable.

\section{CABle Modeling}

We propose to model a the three meters length cable which a $14 \mathrm{~mm}$ length chafed fault located at one meter. The fault can be represented as a impedance discontinuity. Hence, the characteristic impedance $Z_{c}$ of the cable is 50 and the one of the chafing fault $Z_{f}$ is 62.5658 . The value of $Z_{f}$ has been computed according to the electrostatic model proposed in [11]. we decide to build a code in the time domain based on the transmission line theory. The simulated cable is represented in Fig. 1. Hence, the step mesh $d x$ is fixed to $1 \mathrm{~mm}$ while the time step $d t$ is equal to $\frac{d x}{2 c}$ with $c$ the celerity. Indeed, for chaotic signals it is better to choose $d t<\frac{d x}{c}$ using the transmission line theory. The number of iteration is $N t=$ $f\left(\frac{T \max }{d r}\right)$ with, $f$ the function which rounds the proposed ratio to the nearest integer less than or equal to that ratio, and, $T \max \gg \frac{3000 d x}{c}$. Each segment $d x$ is defined as an inductance and a capacitance in parallel. Between $1 \mathrm{~m}$ to 1.014 $\mathrm{m}$ the inductance is defined as $L_{f}=\frac{Z_{f}}{c}$ and the capacitance as $C_{f}=\frac{1}{c Z_{f}}$. Outside this interval we have $L=\frac{Z_{c}}{c}$ and $C=$ $\frac{1}{c Z_{c}}$. Therefore, the current and the voltage can be computed for each segment for the injected chaotic signal solving the following equations:

$$
\begin{gathered}
L \frac{\partial I}{\partial t}+R I+\frac{\partial V}{\partial x}=0, \\
C \frac{\partial V}{\partial t}+\frac{\partial I}{\partial x}=0 .
\end{gathered}
$$

Then, the correlation between the injected chaotic signal and the obtained signal must be performed. Finally, we do the convolution between the right-side of this signal and a Gaussian signal. This step allows us to filter our signal and in order to obtain a smooth reflected signal.

\section{FIRST CHAOTIC SIGNAL : COMBINATION OF LOGISTIC MAP AND BERNOULLI MAP}

\section{A. Definition}

The logistic map is a chaotic second-order polynomial using non-linear dynamical equations defined by the following equation:

$$
x_{m}+1=k x_{m}\left(1-x_{m}\right) \text { with } m=1,2, \ldots, M,
$$

and, with $k \in[0,4]$ and $\mathbf{M}$ is the samples number.

In the other hand, the Bernoulli map is defined as follow:

$$
y_{n+1}=\left\{\begin{array}{l}
B y_{n}+0.5 \text { if } y_{n}>0 \\
B y_{n}-0.5 \text { if } y_{n}<0
\end{array},\right.
$$

with $\mathrm{B}$ a constant value.

Hence, combining the both maps using the initial conditions $\left\{x_{0}, y_{m, 1}\right\}$, we obtain the following equation:

$$
y_{m, n+1}=\left\{\begin{array}{l}
B_{m} y_{m, n}+0.5 \text { if } y_{m, n}>0 \\
B_{m} y_{m, n}-0.5 \text { if } y_{m, n}<0
\end{array},\right.
$$

with $n=1,2, \ldots, N$ and $B_{m}=1.4+0.6 x_{m}$.

We note that $y_{m, 1}$ is a random vector defined as follow:

$$
y_{m, 1}=U(0,1)-0.5 \forall m \in[1 . . M] .
$$

This random initialization is important in order to ensure a chaotic behavior for some $\mathrm{k}$ values. Indeed, with a constant initial value the generated signal can be pseudo-periodic using $k=2$ for instance.

Finally, the chaotic signal $\mathrm{S}$ is defined as a vector of size $M \times N$ from $y_{m, n}$. It has been demonstrated in [9] that the proposed definition of this signal ensures a chaotic behavior. Thus, notwithstanding the initial conditions, the proposed chaotic signal can be depicted with two parameters which are the number of samples $M \times N$ and the k parameter value. 
TABLE I

PERFORMANCE INDICATORS IN TERMS OF FAULT DETECTION FOR SEVERAL CHAOTIC SIGNALS WITH DIFFERENT NUMBER OF SAMPLES.

\begin{tabular}{ccccc}
\hline \hline & 20k signal & $40 \mathrm{k}$ signal & $50 \mathrm{k}$ signal & $65 \mathrm{k}$ signal \\
\hline$A_{\text {soft foult }}^{\text {peak }}(\mathrm{V})$ & 0.3264 & 0.3521 & 0.3516 & 0.3411 \\
$\sigma_{[0,0.95]}(\mathrm{V})$ & 0.0389 & 0.0348 & 0.0323 & 0.0310 \\
$\Delta(\mathrm{dB})$ & 18.4866 & 20.1130 & 20.7277 & 20.8409 \\
\hline \hline
\end{tabular}

\section{B. Sensitivity analysis}

First, a parametric study on the samples number is performed in terms of fault detection. Four chaotic signals are compared; 1) a 20000 samples signal noted 20k signal, 2) a 40000 samples signal noted 40k signal, 3) a 50000 samples signal noted 50k signal, and, 4) a 65000 samples signal noted $65 \mathrm{k}$ signal. For each signal, $N$ is fixed to 100 while $M$ takes the value $200,400,500$ or 650 respectively for the $20 \mathrm{k}$ signal, 40k signal, 50 signal and $65 \mathrm{k}$ signal. These generated signals using $x_{0}=0.1$ and $k=3.9$ are illustrated in Fig. 2 . We note that the same $y_{m, 1}$ initial vector is used for each generated signal in order to be consistent for comparison.

Then, each signal is the injected voltage $V_{g}$ of Fig. 1 and the reflected signal is computed using the iterative procedure presented in the section II. The obtained reflected signals are illustrated in Fig. 3. It appears clearly the peak related to the soft fault at one meter and the one related to the "hard fault" at three meters. It seems that the peak-to-peak amplitude related to the soft fault at one meter increases with the number of samples of the injected signal. Moreover, the noise level seems to decrease with the number of samples of the injected signal. In order to quantify these assumptions, we define an indicator as follow:

$$
\Delta=20 \log \left(\frac{A_{\text {soft fault }}^{\text {peak to }}}{\sigma_{[0,0.95]}}\right)
$$

with, $A_{\text {soft fault }}^{\text {peak the }}$ the peak-to-peak amplitude related to the soft fault and $\sigma_{[0,0.95]}$ the standard deviation on the interval [0, $0.95] \mathrm{m}$. Thus, the indicator $\Delta$ can be understand as a signal to noise ratio which is hard to define for a chaotic signal because of its own structure. The higher the indicator $\Delta$ is, the better the soft fault detection is. For instance, we can consider the fault detection acceptable while $\Delta>6 \mathrm{~dB}$ which lead to a signal more than twice than the "noise". The results are depicted in Table I. Indeed, the standard deviation decreases with the increase of the number of samples of the injected signal. However, the peak-to-peak amplitude related to the soft fault is higher for the 40k signal than for the 50k signal or $65 \mathrm{k}$ signal. Nevertheless, the "performance indicator" $\Delta$ can be considered better increasing the number of samples according to the results of Table I.

In order to be more accurate, a sensitivity analysis is carried out for 601 injected signals from 20000 samples up to 80000 samples with $x_{0}=0.1$ and 601 injected signals from 20000 samples up to 80000 samples with $x_{0}=0.3$. As previously, the same $y_{m, 1}$ initial vector is used for each
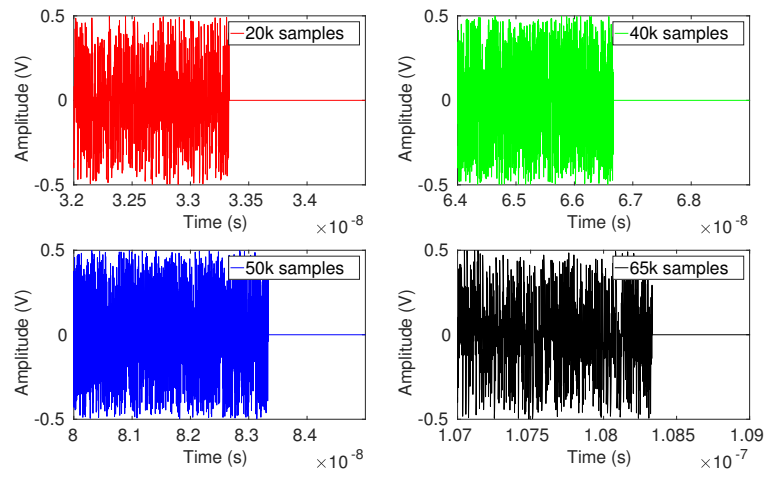

Fig. 2. Zoom on the different generated chaotic signals

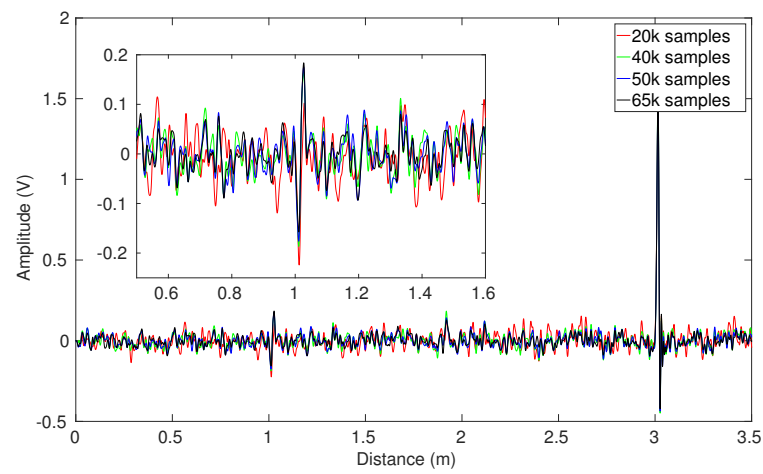

Fig. 3. Reflected signals for several chaotic signals with different number of samples. The inset is a zoom of the signals on the interval $[0.5,1.6] \mathrm{m}$

generated signal in order to be consistent for comparison. Fig. 1 shows the performance indicator $\Delta$ for each signal. We note that, as expected by chaotic signal definition, the results are sensitive to the initial conditions. However, the trend is the same; the performance indicator is increasing with the sample numbers even if the curves are not strictly increasing. From a certain number of sample, which varies according to initial conditions, the performance indicator rise can be considered as insignificant. This result is in agreement with the proposed fitting equation of the noise level given in [10]. Indeed, increasing the samples number, we increase the information number and as a consequence we enhance the correlation of the injected and reflected signal.

Finally, the sensitivity analysis on the $k$ parameter of the proposed chaotic signal is performed. We take 1) the $65 \mathrm{k}$ signal with $x_{0}=0.1$ as initial condition and a random vector $y_{m, 1}$ as first seed for $41 k$ values from 1 to 4 and 2) the same signal with another random vector $y_{m, 1}$ as second seed for the same $k$ values. The performance indicator and the amplitude peak-to-peak related to the soft fault are illustrated in Fig. 5. As previously, we note that the performance indicator is sensitive to the initial conditions even if the trend is relatively similar. Hence, this indicator is slightly sensitive to the $k$ value. However, this sensitivity can be considered as insignificant as well as $\Delta \gg 6 \mathrm{~dB}$. Furthermore, the amplitude 


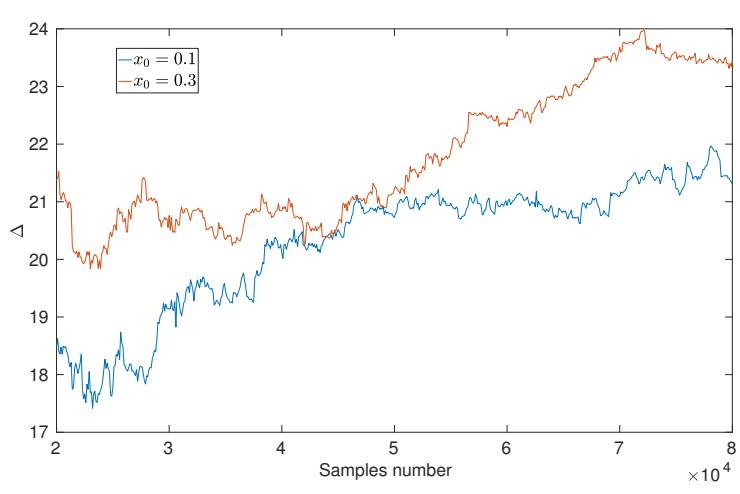

Fig. 4. Performance indicator versus samples number of the injected chaotic signal for two different initial conditions

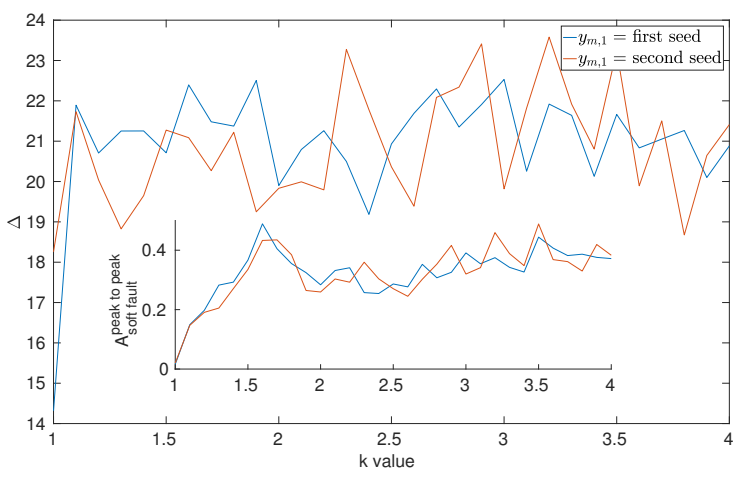

Fig. 5. Performance indicator and versus the $k$ value of the injected chaotic signal for two different initial seeds. The inset show the amplitude peak-topeak related to the soft fault versus the $k$ value of the injected chaotic signal for each seed.

peak-to-peak is interesting in order to comment the dynamic of the problem. Indeed, for measurement point of view, the measurement dynamic could be an issue. The results shows than, except for $k=1$, the amplitude peak-to-peak value is higher than $0.1 \mathrm{~V}$.

To conclude, the proposed chaotic signal seems to be interesting in terms of fault detection whatever the parameters. However, it has been shown than it should be better to increase the number of samples of the injected signal in order to reduce the noise level, and, as a consequence, to improve the fault detection.

\section{SECOND CHAOTIC SIGNAL : THE LORENZ MAP}

\section{A. Definition}

The second chaotic signal used is the Lorenz chaotic signal. Edward Lorenz discovered a three nonlinear coupled ordinary differential equations called the Lorenz model. The dynamics of the Lorenz chaotic system can be described in the equations:

$$
\begin{gathered}
\frac{\partial x}{\partial t}=\sigma(y(t)-x(t)) \\
\frac{\partial y}{\partial t}=r x(t)-y(t)-x(t) z(t),
\end{gathered}
$$
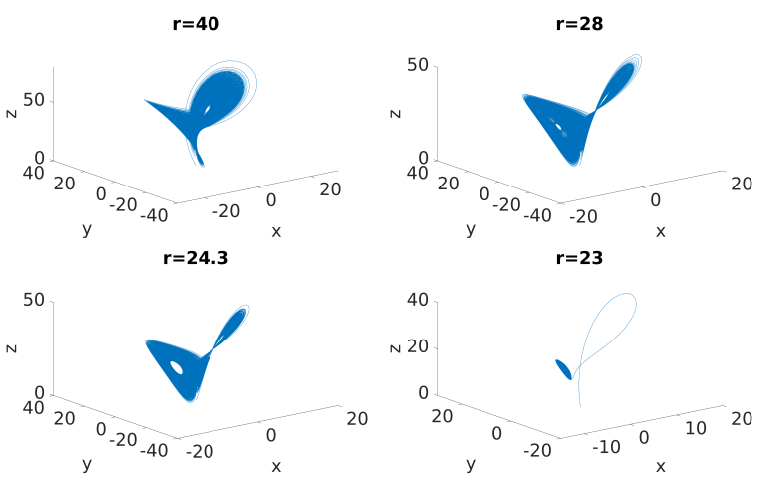

Fig. 6. Lorenz attractors for different $r$ values
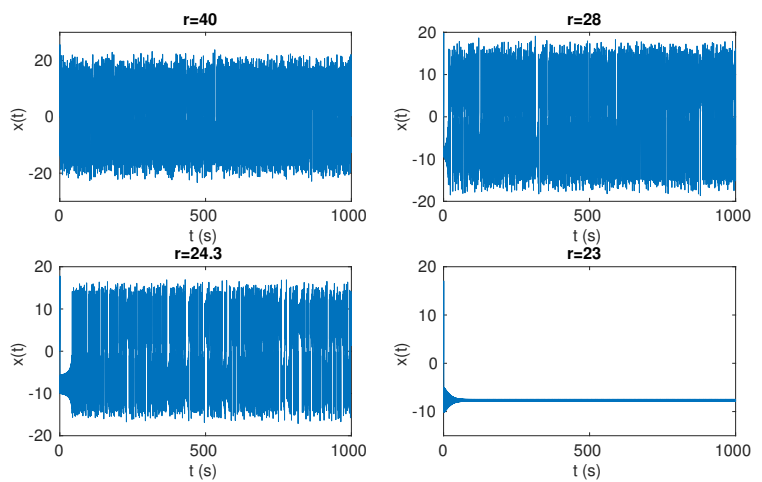

Fig. 7. Lorenz attractors for different $r$ values

$$
\frac{\partial z}{\partial t}=-b z(t)+x(t) y(t)
$$

with $\sigma, b$ and $r$ three parameters. Usually, $\sigma=10, b=\frac{8}{3}$ and $r$ is variable. Nevertheless, to obtain a chaotic behavior the $r$ parameter value must be higher than 24.3. Indeed, according to the Lorenz attractor plots in Fig. 6, we note that under this threshold value the attractor converges to one value. As a consequence, for instance, the signal $x(t)$ begin pseudoperiodic as shown in Fig. 7. We note also that the chaotic behavior can have some lags for $r=28$ for example. It means that the signal is pseudo-periodic in the first 18 seconds, then, the signal has a chaotic behavior. Increasing the $r$ value this lag is reduced. At $r=40$, the signal has a chaotic behavior since zero second.

Moreover, the discussion about the threshold value and the time lag is also related to the numerical solver of the Lorenz equations. The previous results (Fig. 6 and Fig. 7) has been produced solving these equations with a 4th order Runge-Kutta algorithm. For instance, using the forward Euler method leads to more important errors and modifies the threshold value [11]

\section{B. Sensitivity analysis}

As previosuly, a sensitivity analysis is carried out on the samples number for two different sets of initial conditions which are $\left\{t_{0}=0, x_{0}=0.1, y_{0}=0.1, z_{0}=0.1\right\}$ and 


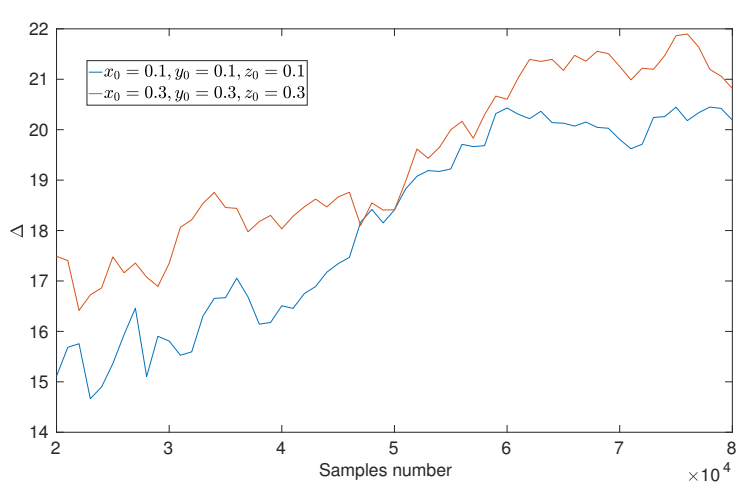

Fig. 8. Performance indicator versus samples number of the injected chaotic signal for two different sets of initial conditions

$\left\{t_{0}=0, x_{0}=0.3, y_{0}=0.3, z_{0}=0.3\right\}$. Hence, we analyze the reflected signal from an injected signal $x(t)$ with $\sigma=10$, $b=\frac{8}{3}$ and $r=40$ as parameter. The injected signal is solved with the 4th order Runge-Kutta solver, normalized between $+0.5 \mathrm{~V}$ and $-0.5 \mathrm{~V}$ and compressed in the time scale with the same $d t$ than the used signals in the previous section. The sensitivity analysis results are illustrated in Fig. 8. The same comments as the ones made in section III can be made. Even if the results are sensitive to initial conditions, the higher the sample number, the better the soft fault detection. From a certain number of sample, which can vary according to initial conditions, the performance indicator rise can be considered as insignificant. Hence, in the following each studied signal has 65000 samples with $\left\{t_{0}=0, x_{0}=0.1, y_{0}=0.1, z_{0}=0.1\right\}$ as initial conditions.

Thus, another sensitivity analysis on the $r$ value is performed for both sets of initial conditions. Fig. 9 presents the results in terms of performance indicator and amplitude peakto-peak related to the soft fault. We note important variation of the performance indicator with the $r$ value, for instance around $7 \mathrm{~dB}$ as maximum difference with $\left\{t_{0}=0, x_{0}=\right.$ $\left.0.1, y_{0}=0.1, z_{0}=0.1\right\}$ as initial conditions. The variation of the performance indicator with the initial conditions is also highlighted. However, using the Lorenz map as a chaotic signal, the soft fault detection could be performed because of $\Delta \gg 6 \mathrm{~dB}$. In a measurement point of view, we note that the Lorenz signal leads to higher amplitudes peak-to-peak related to the soft fault in comparison with the ones produces with the combination of Logistic map and Bernoulli map. Nevertheless, according to both performance indicator, the produced noise is also higher (around the same factor) using the Lorenz signal.

\section{PERFORMANCE COMPARISON}

In the previous sections, we have seen in particular that the proposed chaotic signals are sensitive to initial conditions and their own parameters. Therefore, the performance comparison between both signals in terms of fault detection requires a statistical approach. As a consequence, we perform 1) 30 simulations with a random initial conditions $x_{0} \in[0,1]$ for each $k$ value of the first chaotic signal (noted Bernoulli signal),
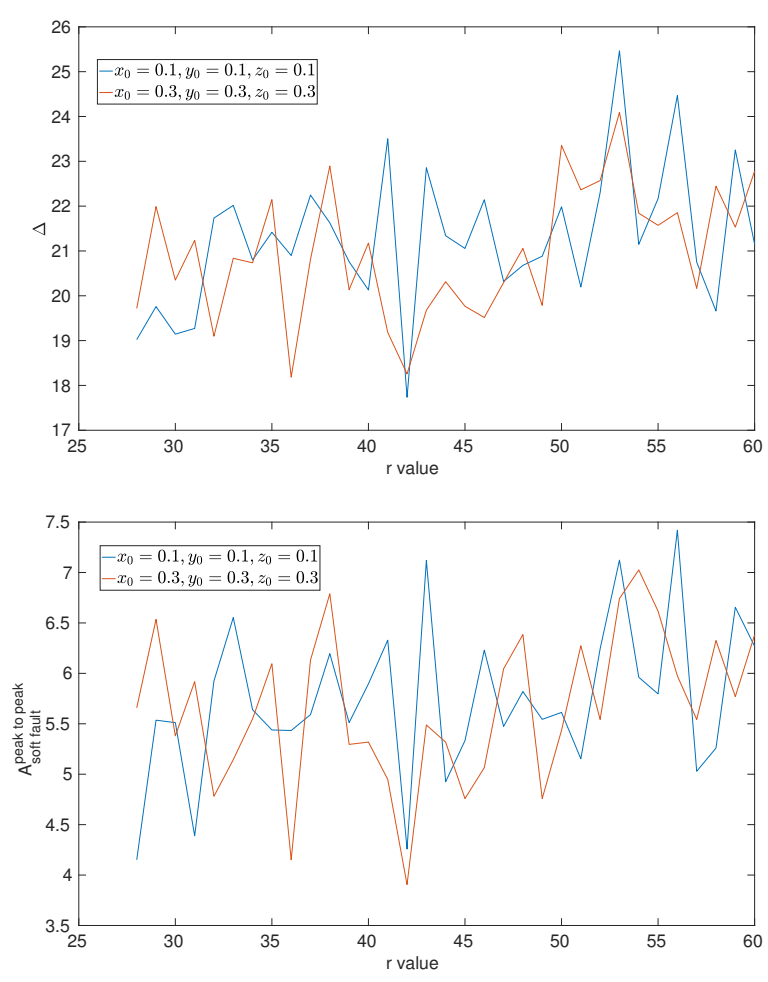

Fig. 9. Performance indicator (at top) and amplitude peak-to-peak related to the soft fault (at bottom) versus the $r$ value of the injected chaotic signal for two different sets of initial conditions

and, 2) 30 simulations with a random set of initial conditions $\left\{x_{0} \in[0,1], y_{0} \in[0,1], z_{0} \in[0,1]\right\}$ for each $r$ value of the second chaotic signal (noted Lorenz signal). Both signals are built with 65000 samples. For each parameter value, the mean of the performance indicator $\tilde{\delta}$ is computed as well as the standard deviation $\sigma$ and its mean $\tilde{\sigma}$. Moreover, the mean performance indicator $\bar{\Delta}$ and the mean standard deviation $\bar{\sigma}$ are computed as follow:

$$
\begin{aligned}
& \bar{\Delta}=\frac{1}{p} \sum_{1}^{p} \tilde{\delta}, \\
& \bar{\sigma}=\frac{1}{p} \sum_{1}^{p} \tilde{\sigma},
\end{aligned}
$$

with $p$ the samples number of the parameters $r$ or $k$ which is equal to 31 .

Fig. 10 presents the mean performance indicators $\tilde{\delta}$ for both signals while the error bars represents the standard deviation for each parameter value. We note that, overall, the mean performance indicator is increasing with the $r$ value for the Lorenz signal in contrast with the Bernoulli signal which can presents an important variation with the $k$ value. Furthermore, the Lorenz signal is more sensitive to initial conditions than the Bernoulli signal. Overall, the Bernoulli signal is more reliable for the soft fault detection with a better mean performance indicator and less variation than the Lorenz signal according to 


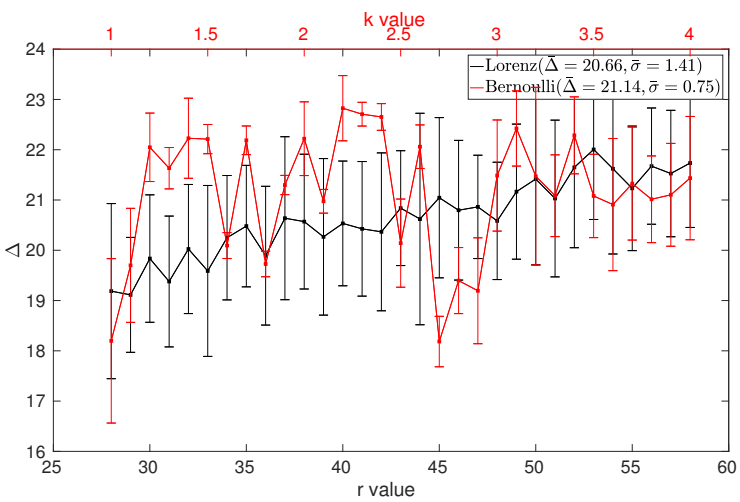

Fig. 10. Mean performance indicator versus the parameter value of each injected chaotic signal for 30 different sets of initial conditions. The error bars represents the standard deviation for each 30 different sets of initial conditions.

$\bar{\Delta}$ and $\bar{\sigma}$. However, the $k$ value must be "well chosen" in order to obtain a relative optimum of $\Delta$. Moreover, increasing the $r$ value of the Lorenz signal could lead to better results even if the variation with initial conditions will be yet important in our opinion. To conclude, the study shows that the Bernoulli signal seems to be a more reliable choice than the Lorenz signal for the soft fault detection while the $k$ value is well chosen. Nevertheless, both signals propose good performances in terms of soft fault detection.

\section{CONCLUSION}

We had proposed in this article a simulation analysis with two signals 1) a combination of the logistic map and Bernoulli map and 2) a Lorenz signal for the soft fault detection in cables. A sensitivity analysis has lead to increase the sample number of each chaotic signal in order to obtain better performances. This analysis has also highlighted the high sensitivity to initial conditions and the parameter value of each signal. Hence, a statistical study has been carried out in order to compare the both signals. The results demonstrates a better reliability for the combination of the logistic map and Bernoulli map than the Lorenz signal, in particular in terms of sensitivity about initial conditions. However, it has been shown in this article that the both signals could be used as they present very good performances in terms of soft fault detection. In the future, their performances and limitation with the impedance value related to the soft fault will be studied as well as their performances in a noisy environment. Moreover, it could be interesting to study their relevance for complex wire network and their ability in measurement.

\section{REFERENCES}

[1] F. Auzanneau, "Wire troubleshooting and diagnosis: Review and perspectives," Progress In Electromagnetics Research, vol. 49, pp. 253-279, 2013.

[2] C. Furse, Y. C. Chung, R. Dangol, M. Nielsen, G. Mabey, and R. Woodward, "Frequency-domain reflectometry for on-board testing of aging aircraft wiring," IEEE Transactions on Electromagnetic Compatibility, vol. 45 , no. 2 , pp. 306-315, 2003.
[3] C. S. Hood and C. Ji, "Proactive network-fault detection [telecommunications]," IEEE Transactions on reliability, vol. 46, no. 3, pp. 333-341, 1997.

[4] I. Bzikha, C. Guiffaut, and A. Reineix, "Time-frequency analysis for wiring soft faults detection," in 2019 International Symposium on Electromagnetic Compatibility - EMC EUROPE, Sep. 2019, pp. 481485.

[5] L. El Sahmarany, L. Berry, N. Ravot, F. Auzanneau, and P. Bonnet, "Time reversal for soft faults diagnosis in wire networks," Progress In Electromagnetics Research, vol. 31, pp. 45-58, 2013.

[6] F. Auzanneau, M. Olivas, and N. Ravot, "A simple and accurate model for wire diagnosis using reflectometry," in Piers Proceedings, 2007, pp. 232-236.

[7] F. Loëte, S. Noël, R. Meyer, M. Olivas, F. Auzanneau, and D. Chandon, "Feasability of the detection of vibration induced faults in connectors by reflectometry," in 24th International Conference on Electrical Contacts. Saint-Malo, 2008, pp. 440-443.

[8] H. Xu, B. Wang, J. Li, A. Wang, and Y. Wang, "Location of wire faults using chaotic signal generated by an improved colpitts oscillator,' International Journal of Bifurcation and Chaos, vol. 24, no. 04, p. 1450053, 2014.

[9] F. Auzanneau, N. Ravot, and L. Incarbone, "Chaos time domain reflectometry for online defect detection in noisy wired networks," IEEE Sensors Journal, vol. 16, no. 22, pp. 8027-8034, 2016.

[10] F. Auzanneau, "Chaos time-domain reflectometry for distributed diagnosis of complex topology wired networks," Electronics Letters, vol. 52 no. 4, pp. 280-281, 2016.

[11] I. Bzikha, "Comparison and development of advanced wiring fault detected methods on coaxial cables," Ph.D. dissertation, University of Limoges, 123 avenue Albert Thomas 87060 Limoges, 2019 